\section{POS1133 RELIABILITY OF CONVENTIONAL RADIOGRAPHY OF THE KNEE FOR THE ASSESSMENT OF CHONDROCALCINOSIS: AN ANCILLARY STUDY OF THE OMERACT ULTRASOUND - CPPD GROUP}

S. Sirotti ${ }^{1}$, F. Becce ${ }^{2}$, L. M. Sconfienza ${ }^{3}$, C. Pineda ${ }^{4}$, M. Gutierrez ${ }^{4}$, T. Serban ${ }^{5}$, D. Maccarter ${ }^{6}$, A. Adinolfi ${ }^{7}$, E. Naredo ${ }^{8}$, A. Scanu ${ }^{9}$, I. Möller ${ }^{10}$, P. Sarzi-Puttini ${ }^{1}$, A. Abhishek ${ }^{11}$, H. Choi ${ }^{12}$, N. Dalbeth ${ }^{13}$, S. Tedeschi ${ }^{14}$, M. A. D'agostino ${ }^{15}$, H. Keen ${ }^{16}$, L. Terslev ${ }^{17}$, A. lagnocco ${ }^{18}$, G. Filippou ${ }^{1}$ on behalf of OMERACT Ultrasound working group, CPPD subgroup. ${ }^{1}$ Sacco Hospital, Rheumatology Department, Milano, Italy; ${ }^{2}$ Lausanne University Hospital, Department of Diagnostic and Interventional Radiology, Lausanne, Switzerland; ${ }^{3}$ University of Milan, Department of Biomedical Sciences for Health, Milano, Italy; ${ }^{4}$ National Rehabilitation Institute, Division of Musculoskeletal and Rheumatic Diseases, Ciudad de México, Mexico; ${ }^{5}$ Hospital La Colletta, Rheumatology Department, Arenzano, Italy; ${ }^{6}$ North Valley Hospital, Rheumatology Department, Whitefish, United States of America; ${ }^{7}$ Niguarda, Rheumatology Unit, Milan, Italy; ${ }^{8} \mathrm{Hospital}$ Universitario Fundación Jiménez Díaz, Department of Rheumatology and Joint and Bone Research Unit, Madrid, Spain; ${ }^{9}$ University of Padua, Department of Medicine-DIMED, Rheumatology Unit, Padova, Italy; ${ }^{10}$ Instituto Poal de Reumatologia, Rheumatology, Barcelona, Spain; ${ }^{11}$ University of Nottingham, Academic Rheumatology, Nottingham, United Kingdom; ${ }^{12}$ Massachusetts General Hospital, Division of Rheumatology, Allergy, and Immunology, Department of Medicine, Boston, United States of America; ${ }^{13}$ The University of Auckland, Bone and Joint Research Group, Department of Medicine, Faculty of Medical and Health Sciences, Auckland, New Zealand; ${ }^{14}$ Brigham And Women's Hospital, Division of Rheumatology, Inflammation and Immunity, Boston, United States of America; ${ }^{15}$ Agostino Gemelli University Policlinic, Rheumatology Department, Roma, Italy; ${ }^{16}$ The University of Western Australia, School of Medicine, Murdoch, Perth, Australia; ${ }^{17}$ Rigshospitalet, Center for Rheumatology and Spine Diseases, København, Denmark; ${ }^{18}$ University of Turin, Academic Rheumatology Centre, Department of Clinical and Biological Sciences, Torino, Italy

Background: Conventional Radiography (CR) has been widely used in the assessment of knee chondrocalcinosis (CC) and is still considered one of the most important diagnostic methods for the diagnosis. However, there are no studies on the reliability of $\mathrm{CR}$ for CC.

Objectives: To assess the reliability of $\mathrm{CR}$ of the knee in the assessment of chondrocalcinosis (CC).

Methods: This is an ancillary study of the Criterion Validity of Ultrasound in Calcium Pyrophosphate Deposition Disease (CPPD) study [1]. Consecutive patients with knee osteoarthritis $(\mathrm{OA})$ that were planned for total knee replacement surgery were enrolled in 4 centres from Romania, Italy, USA and Mexico. All patients underwent $\mathrm{CR}$ of the knees taken maximum 6 months before surgery, in posterior-anterior weight baring and lateral projections. DICOM files of the radiographs were retrieved, anonymised and read independently by two musculoskeletal radiologists with experience in microcrystalline arthropathies. Each reader performed a second evaluation 3 weeks after the first one to calculate the inter- and intra-reader agreement. For each patient a dichotomic score was assigned (absence/presence of CC) at the level of the medial and lateral menisci, tibiofemoral hyaline cartilage, quadriceps and patella tendons, synovial membrane/joint capsule. The definitions of the ACR/EULAR taskforce for identification of CPPD in conventional radiography were used in this study [paper in preparation]. According to these definitions CPPD in CR appears as "linear or punctate opacities in the region of fibro- or hyaline articular cartilage/synovial membrane or joint capsule/within tendons or entheses that are distinct from denser, nummular radio-opaque deposits due to basic calcium phosphate deposition". Cohen's kappa was used to calculate the agreement between the two readers.

Results: We enrolled 33 patients with knee OA (60.6\% female, mean age $69 y 0$ \pm 8 ). The kappa values of the inter-reader and intra-reader agreement in the various sites of the knee are indicated in Table 1. Inter-reader agreement was substantial at the level of both menisci but only moderate or fair at the other sites of assessment. This had a negative impact on the overall evaluation of the knee joint that proved to be unreliable ( $k$ of 0.16 - none to slight agreement) if all anatomical structures are included for assessment, and moderately reliable (kappa 0.41 ) when both menisci and hyaline cartilage are considered. On the other hand, intra-reader kappa values were substantial or higher in all sites (except for synovial membrane/joint capsule for one reader). The striking difference of the intra-reader compared to the inter-reader kappa values, highlight a different interpretation and application of the definitions used for most of the sites with the exception of the menisci.
Table 1. kappa values for intra- and inter-reader agreement. Values from $0.01-0.20$ are considered as none to slight agreement, $0.21-0.40$ as fair, $0.41-0.60$ as moderate, $0.61-0.80$ as substantial, and $0.81-1.00$ as almost perfect agreement.

Medial Lateral Hyaline Quadriceps Patellar Capsule/ Menisci + Entire meniscus menis- cartilage tendon tendon synovia cartilage joint cus

\begin{tabular}{|c|c|c|c|c|c|c|c|c|}
\hline Inter-reader & 0.67 & 0.71 & 0.34 & 0.47 & NA & 0.37 & 0.40 & 0.17 \\
\hline $\begin{array}{l}\text { Intra-reader } \\
1^{\text {st }} \\
\text { assessor }\end{array}$ & 0.67 & 0.90 & 0.84 & 0.65 & $\begin{array}{c}\mathrm{NA} \\
\text { (insufficient } \\
\text { number of } \\
\text { categories) }\end{array}$ & 0.53 & 0.71 & 0.76 \\
\hline $\begin{array}{l}\text { Intra-reader } \\
2^{\text {nd }} \\
\text { assessor }\end{array}$ & 1 & 0.80 & 1 & 1 & 1 & 0.91 & 0.86 & 0.94 \\
\hline
\end{tabular}

Conclusion: CR has been extensively used for diagnosis of OA and CPPD. The results of our study raise some concerns on the reliability of $\mathrm{CR}$ in identification of CPPD. Assessment of calcium crystals at the menisci level should be used for identification of $\mathrm{CC}$ as other sites of the knee seem to present low reliability. REFERENCES:

[1] Filippou $\mathrm{G}$ et al. Criterion validity of ultrasound in the identification of calcium pyrophosphate crystal deposits at the knee: an OMERACT ultrasound study. Ann Rheum Dis 2020. doi:10.1136/annrheumdis-2020-217998

Disclosure of Interests: None declared.

DOI: 10.1136/annrheumdis-2021-eular.1438

\section{POS1134 COMPARATIVE ANALYSIS OF THE CLINICAL AND LABORATORY PROPERTIES OF GOUT, OSTEOARTHRITIS, AND CALCIUM PYROPHOSPHATE DEPOSITION DISEASE}

D. Kravchenko ${ }^{1}$, R. Bergner ${ }^{2}$, C. Behning ${ }^{3}$, V. Schäfer ${ }^{4} .{ }^{1}$ University Hospital Bonn, Department of Diagnostic and Interventional Radiology, Bonn, Germany; ${ }^{2}$ Klinikum Ludwigshafen, Medical Clinic A: Clinic for Internal Medicine, Hematology, Nephrology, Infektiology and Rheumatology, Ludwigshafen, Germany; ${ }^{3}$ University Hospital Bonn, Institute for Medical Biometrics, Informatics and Epidemiology (IMBIE), Bonn, Germany; ${ }^{4}$ University Hospital Bonn, Clinic of Internal Medicine III, Hematology, Oncology, Rheumatology and Clinical Immunology, Bonn, Germany

Background: The clinical differentiation between gout, osteoarthritis (OA), and calcium pyrophosphate deposition disease (CPPD) still remains a hurdle in daily practice without imaging or arthrocentesis. Although a plethora of clinical data exists, reliable predictor biomarkers for all but gout are still missing.

Objectives: To explore an association between common physical examination, ultrasound and laboratory findings and gout, OA, and CPPD, which can in turn provide reliable diagnostic predictions.

Methods: 277 patients were retrospectively analysed using ANOVA with Scheffe's post hoc tests and conditional inference trees regarding biomarkers such as age, sex, body mass index, hypertension, renal status, cumulative affected joint size, number of afflicted joints, double contour sign, intracartiliginous double contour sign, degree of vascularization on ultrasound (DoV), uric acid, erythrocyte sedimentation rate (ESR), C-reactive protein (CRP), ferritin, and leucocyte count Simple linear regressions were carried out to explore associations between increased inflammatory parameters and the above-mentioned biomarkers. Statistically significant associations were defined as $p$ values $<0.05$.

Results: The male sex was associated with gout ( $p$ value $<0.05$ vs CPPD and $<0.05$ vs OA). OA affected younger patients than CPPD (mean 64.5 vs 73.1 years, $p<0.05)$. Hypertension was correlated with gout $(p<0.05)$ and CPPD ( $p$ $<0.05)$, impaired renal status with gout when compared to $O A(p<0.05)$ but not compared to CPPD ( $p$ 0.21). A higher number of involved joints was associated with gout (mean 2.2 joints) compared to OA $(1.0, p<0.05)$ and CPPD (1.6, p 0.01). The double contour sign was not able to differentiate gout and CPPD with a sensitivity/specificity of $71 \% / 55 \%$ for gout and $59 \% / 39 \%$ for CPPD. The intracartilaginous double contour sign was specific for CPPD (99\%) but with a low sensitivity of $26 \%$. DoV was significantly associated with gout (vs OA, $p<0.05$ ) and CPPD (vs OA, $p<0.05$ ). Unsurprisingly, uric acid was associated with gout while ESR and CRP were increased in gout and CPPD, but not in OA. Some associations were statistically significant but arguably clinically unimportant. Conditional inference trees were able to exclude OA (specificity 97.5\%) and CPPD (specificity $94.0 \%$ ) as possible differentials based on just uric acid, CRP, hypertension, 